\title{
Descripción de dos nuevas especies y nuevos registros del género Epilachna Chevro- lat (Coleoptera: Coccinellidae) en el Perú
}

\section{Description of two new species and new records of the genus Epilachna Chevrolat (Coleop- tera: Coccinellidae) in Peru}

\section{Abdhiel Bustamante *, Anahí Oróz, Araseli Elme, Edgar Marquina y Erick Yábar}

\author{
Universidad Nacional San Antonio Abad del Cusco UNSAAC, Escuela Profesional de Biología, Colección Entomológica (CEUC). Av. de la Cultura, 733 \\ Cusco, Cusco, Perú. \\ Abdhiel Bustamante: http://orcid.org/0000-0001-8120-1274; Email: abdhiel77@gmail.com; \\ Anahi Oroz: http://orcid.org/0000-0003-1833-4204; Email: anahijeannette@yahoo.com \\ Araseli Elme: http://orcid.org/0000-0002-4621-4931; Email: 120338@unsaac.edu.pe; \\ Edgar Marquina: http://orcid.org/0000-0001-9778-3360; Email: 091689@unsaac.edu.pe; \\ Erick Yábar: http://orcid.org/0000-0003-2389-4417; Email: yabarlandae@gmail.com \\ *Autor para correspondencia
}

\begin{abstract}
Resumen
Epilachna lucianae n. sp. y Epilachna matei n.sp. son descritas en base a ejemplares colectados en las regiones peruanas de Huánuco y Cusco; se proveen además nuevos registros de tres especies del género Epilachna Chevrolat para el Perú.

Palabras clave: Epilachnini; Epilachna; fitófagos; neotropico.

\section{Abstract}

Epilachna lucianae n. sp. and Epilachna matei n. sp. are described using specimens collected in the Peruvian regions of Huanuco and Cusco; new records are also provided for some species of the genus Epilachna Chevrolat for Peru.
\end{abstract}

Keywords: Epilachnini; Epilachna; phytophagous; neotropics.

Publicación registrada en Zoobank/ZooBank article registered:

LSID urn:Isid:zoobank.org:pub:F8AD7B07-7679-4BE1-987B-4B383A3B65D3

Acto nomenclatural/nomenclatural act:

1. Epilachna lucianae Bustamante Navarrete, Oróz, Elme-Tumpay, Marquina-Montesinos \& Yabar-Landa, 2018 LSID urn:Isid:zoobank.org:act:E921F386-EBFB-4FBD-A6A7-8B36723F2E8F

2. Epilachna matei Bustamante Navarrete, Oróz, Elme-Tumpay, Marquina-Montesinos \& Yabar-Landa, 2018 LSIDurn:Isid:zoobank.org:act:1D6893BC-BFEC-42A3-A54D-0B702D017726

\section{Citación:}

Bustamante A., A. Oroz, A. Elme, E. Marquina y E. Yábar. 2018. Descripción de dos nuevas especies y nuevos registros del género Epilachna Chevrolat (Coleoptera: Coccinellidae) en el Perú. Revista peruana de biología 25(1): 003 - 010 (Febrero 2018). doi: http://dx.doi.org/10.15381/ rpb.v25i1.13813

$\begin{array}{ll}\text { Presentado: } & 06 / 09 / 2017 \\ \text { Aceptado: } & 04 / 01 / 2018\end{array}$

$\begin{array}{ll}\text { Aceptado: } & 04 / 01 / 2018 \\ \text { Publicado online: } 27 / 02 / 2018\end{array}$

Información sobre los autores:

$A B$ : preparación de ejemplares e identificacion de ejemplares. $A B, A O$ fotografiado y redacción de textos. $E M$ : preparación de ejemplares, fotografiado. $A E, E Y$ : redacción de textos. $A B, A O, A E, E M, E Y$ : revisaron y aprobaron el manuscrito.

Los autores no incurren en conflictos de intereses.

Journal home page: http://revistasinvestigacion.unmsm.edu.pe/index.php/rpb/index

(c) Los autores. Este artículo es publicado por la Revista Peruana de Biología de la Facultad de Ciencias Biológicas, Universidad Nacional Mayor de San Marcos. Este es un artículo de acceso abierto, distribuido bajo los términos de la Licencia Creative Commons Atribución-NoComercial-Compartirlgual 4.0 Internacional.(http://creativecommons.org/licenses/by-nc-sa/4.0/), que permite el uso no comercial, distribución y reproducción en cualquier medio, siempre que la obra original sea debidamente citadas. Para uso comercial, por favor póngase en contacto con editor.revperubiol@gmail.com. 


\section{Introducción}

El género Epilachna Chevrolat, 1837, está ubicado dentro de la tribu Epilachnini, y se distribuye en áreas tropicales y subtropicales de Suramérica conteniendo a la mayoría de las especies de la tribu, junto al género Henosepilachna Li (Szawaryn et al., 2015). El género Epilachna, cuyos integrantes son estrictamente herbívoros, fue revisado por Gordon (1975), quien agrupó a sus especies en 34 grupos de afinidad morfológica; posteriormente, el género fue restringido al Nuevo Mundo por Szawaryn et al. (2015), quienes además indicaron la necesidad de redefinir el género, formado por múltiples clados monofileticos. Tomaszewska y Szawaryn (2016), sobre la base de análisis propios y los del trabajo de Szawaryn et al. (2015), redefinieron todos los géneros de Epilachnini, transfiriendo 12 especies de Epilachna a Toxotoma Weise.

La revisión de ejemplares de Epilachnini depositados en la Colección Entomológica de la Universidad Nacional San Antonio Abad del Cusco, Perú, permitió determinar el estado de especies no descritas para dos morfotipos pertenecientes al género Epilachna, los cuales son descritos en la presente contribución; por otro lado, el examen de la colección también permitió establecer como nuevos registros geográficos para el Perú a Epilachna harringtoni Gordon, 1975, Epilachna strictanotata, Gordon 1975 y Epilachna fenestroides Gordon, 1975.

\section{Material y métodos}

Se siguió el criterio taxonómico de Tomaszewska y Szawaryn (2016). Además, se consideró el trabajo de Gordon (1975) para asignar a los nuevos taxones dentro de los grupos de especies Epilachna v-pallidum y Epilachna vittigera.

La terminología de la estructura genital masculina corresponde a la utilizada por Ślipiński (2007). El tratamiento de los aparatos genitales consistió en la disposición del abdomen del adulto en una solución de $\mathrm{KOH}$ al $10 \%$, sometido al calor por un lapso de 5-10 minutos; luego de un lavado con alcohol, el abdomen fue sumergido en agua oxigenada y sometido a calentamiento por espacio de 5 minutos; el abdomen fue lavado luego en agua destilada y la estructura genital fue separada, bajo el microscopio estereoscópico, con ayuda de estiletes. Las partes analizadas fueron fotografiadas y luego conservadas en glicerina dentro de microviales junto a su respectivo ejemplar. Los hábitos fueron fotografiados con enfoque gradual en serie empleando la cámara AxioCam ICc5, montada sobre un microscopio estereoscopio Discovery $\mathrm{V} 20$, mientras que los aparatos genitales fueron fotografiados utilizando un microscopio Novel $\mathrm{N}-800 \mathrm{~m}$ con una cámara Truechrome II; finalmente las fotos seriadas, de hábito y aparatos genitales, fueron combinadas utilizando el software Helicon Focus. Los datos de las etiquetas han sido transcritos literalmente y se ańaden datos faltantes entre corchetes [ ]. Los ejemplares examinados se encuentran depositados en la Colección Entomológica UNSAAC (CEUC), Universidad Nacional San Antonio Abad del Cusco, Perú.

\section{Resultados \\ CLASS: INSECTA \\ ORDER: COLEOPTERA \\ FAMILY: COCCINELLIDAE \\ SUBFAMILY: COCCINELLINAE \\ TRIBU: EPILACHNINI}

Genero: EpILACHNA CheVrolat, 1837

Epilachna Chevrolat in Dejean, 1837: 460. Epilachna sensu stricto Szawaryn et al., 2015: 561.

\section{Epilachna lucianae $\mathbf{n . s p}$.}

(Figuras $01-14,35)$

Taxón ubicado en el grupo de especies Epilachna v-pallidum, por presentar el aparato genital masculino con el lóbulo basal, o guía del pene, fuertemente comprimido de manera lateral y curvado abruptamente hacia arriba hasta el ápice redondeado y por presentar el pene en forma de " $S$ ". El grupo se distribuye principalmente en Bolivia y el sur del Perú con una sola especie en Ecuador (Gordon 1975).

Diagnosis: Epilachna de color negro, presenta élitros de color marrón claro totalmente bordeados de negro; en la parte media de cada élitro está presente una mancha negra alargada aislada, transversa, más cercana al borde elitral que a la sutura. Presenta semejanza con Epilachna cuscoi Gordon,1975 y E. dorsigera Erichson, 1847 por la coloración clara dominante de los élitros, aunque estas dos especies carecen de la mancha negra transversa y de la banda sutural, además de presentar el aparato genital masculino característico del grupo dorsigera. También existen semejanzas con la especie E. abrupta Gorham, 1897, restringida a Costa Rica y Panamá, la cual tiene predominio de la coloración clara en los élitros y una banda transversal que une los bordes lateral y sutural a la mitad del élitro; esta especie presenta el aparato genital masculino característico del grupo $E$. deuterea.

Descripción: Macho: largo total $8 \mathrm{~mm}$, ancho $6 \mathrm{~mm}$, alto 4 $\mathrm{mm}$. Cuerpo oval alargado, dorso moderadamente curvado, con pubescencia densa y corta. Cabeza negra con pubescencia clara, larga y densa. Pronoto negro con pubescencia amarilla densa. Escutelo triangular, negro. Élitros marrón claro, bordeados por una banda delgada negra, más ancha en los ápices elitrales y en el hombro y muy adelgazada entre el húmero y el escutelo, presentando una mancha oscura alargada transversal en la parte media del élitro, ligeramente más cercana al margen lateral que al sutural. Lado inferior completamente negro, incluyendo las epipleuras. Patas negras con pubescencia amarilla, corta, más densa en las tibias y discos tarsales. Cabeza con puntuación fina, espaciada por una distancia mayor a 3 veces el diámetro de cada punto. Distancia intraocular equivalente a 5.5 diámetros oculares en vista dorsal. Pronoto con los márgenes anteriores lateral proyectados hacia adelante, margen anterior ligeramente escotado medialmente. Disco pronotal con puntos finos y densamente distribuidos, separados por una distancia equivalente a 2 diámetros, puntuación más grande en los márgenes elitrales y separadas por una distancia equivalente a 1.5 - 2 diámetros. Disco elitral pon puntuación dual, puntos grandes separados por 2-3 diámetros o más y puntos pequeños separados por 1-2 diámetros. Superficies de metasterno y mesosterno cubiertas de setas cortas, finas y distribuidas irregularmente. Borde posterior del sexto ventrito abdominal moderadamente escotado, hasta un cuarto de su ancho (Figura 07).

Hembra: largo total $10.5 \mathrm{~mm}$, ancho $7 \mathrm{~mm}$, alto $5 \mathrm{~mm}$. Similar al macho, tamaño algo mayor y coloración más clara de los élitros (Figuras 11, 12). Borde posterior del sexto ventrito abdominal fuertemente escotado, hasta dos tercios de su ancho (Figura 08). 

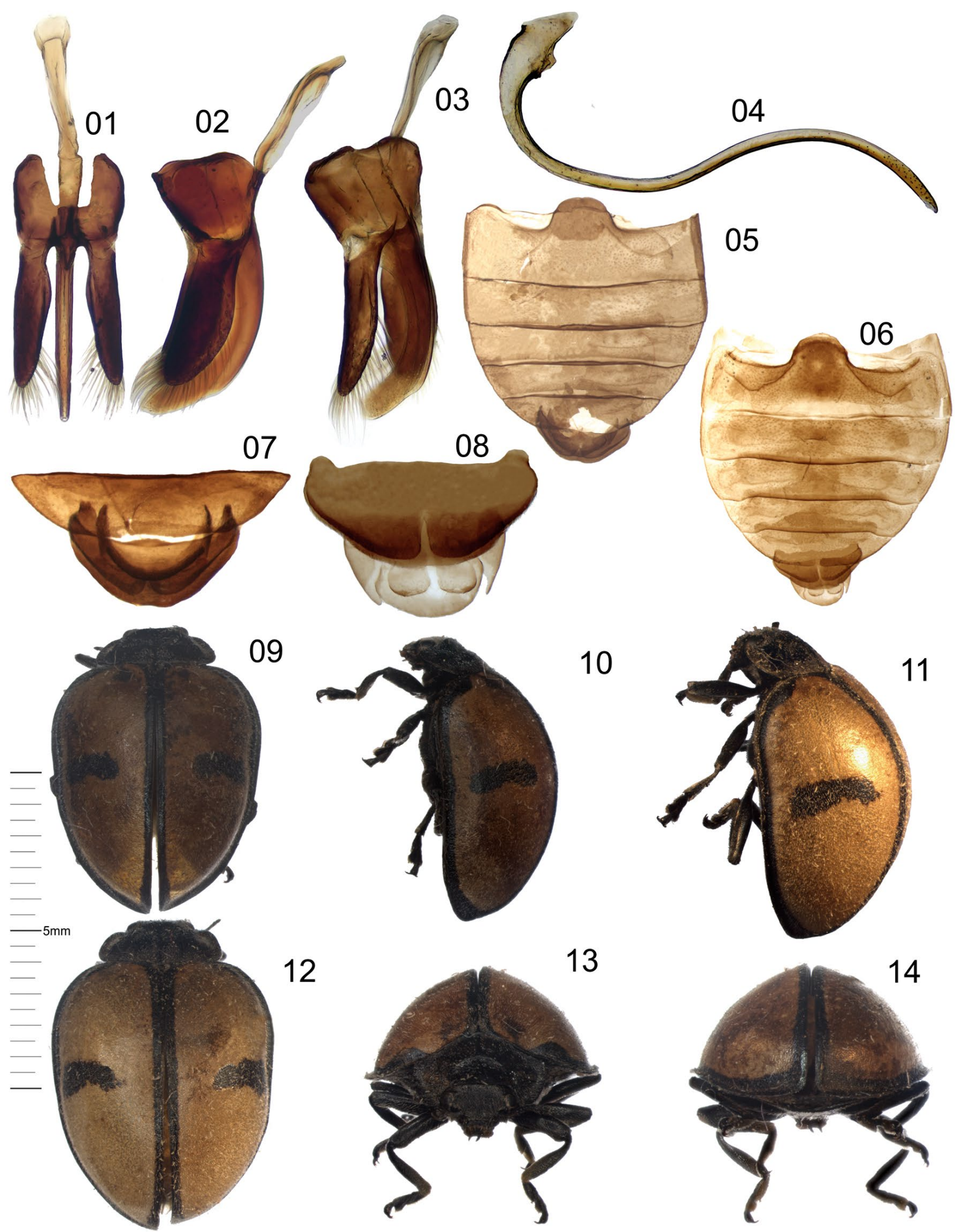

10

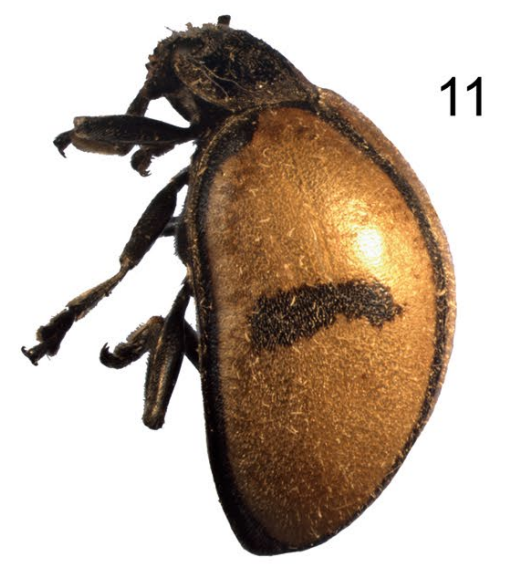

12
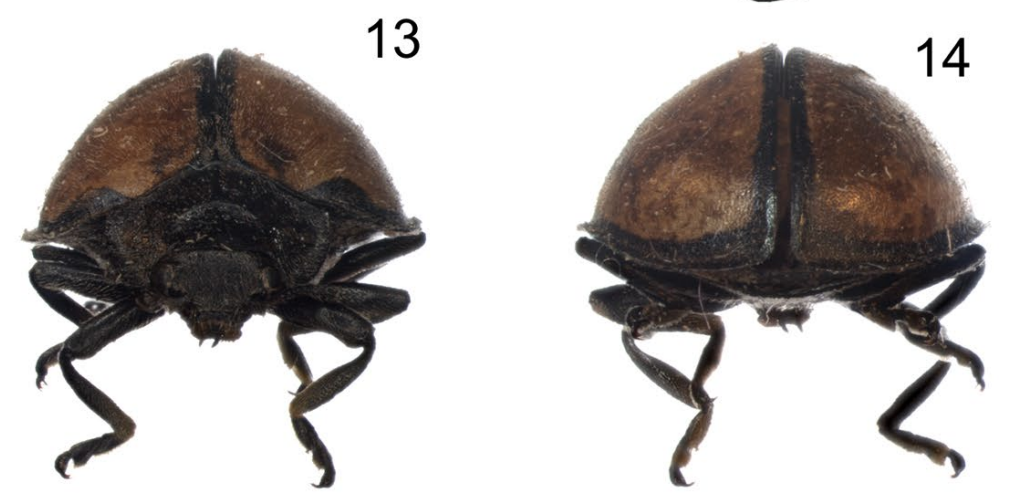

Figuras 01 - 14: Epilachna lucianae n. sp. (01) Tegmen, vista frontal; (02) Tegmen, vista lateral; (03) Tegmen, vista fronto lateral; (04) Pene, vista lateral; (05) Abdomen, macho; (06) Abdomen, hembra; (07) Urosternitos terminales, macho; (08) Placa genital, hembra; (09) Hábito macho, vista dorsal; (10) Hábito macho, vista lateral; (11) Hábito hembra, vista lateral; (12) Hábito hembra, vista dorsal; (13) Hábito macho, vista anterior; (14) Habito macho, vista posterior. 
Aparato genital masculino: tegmen con la guía del pene, o lóbulo basal, comprimido lateralmente y curvado abruptamente hacia arriba, ápice redondeado; parámeros más cortos que la guía del pene, engrosados en su parte media y adelgazados hacia el ápice redondeado, presencia de setas en el tercio apical (Figuras $01,02,03$ ); el pene en forma de $S$, largo, adelgazado, capsula del pene redondeada con una proyección externa, larga y ahusada, y una proyección interna corta, subcuadrangular, ápice redondeado (Figura 04).

Aparato genital femenino: placa genital femenina con los coxitos subtriangulares y el stylus visible, margen apical con pequeńa protuberancia y presencia de setas (figura 8 ). No se estudió la espermateca.

Holotipo: [PERU], Picahuay, 13.05.09 [13 mayo 2009], [distrito de Umari, provincia de Pachitea], [región] Huánuco, 2929m, 18L0382957 UTM 8904344 [954'36.21"S $76^{\circ} 4$ '3.37”O], W. Cosio L. [colector].

Alotipo: (hembra) mismos datos que el holotipo.

Paratipos: dos ejemplares hembra, mismos datos que el holotipo.

Variación: largo total $8-11 \mathrm{~mm}$, ancho $6-8 \mathrm{~mm}$, alto 4 $-6 \mathrm{~mm}$. La mancha digitiforme del élitro varía en grosor, pero nunca llega a tocar los bordes del élitro (figura 11).

Distribución: distrito de Umari, provincia de Pachitea, región Huánuco, Perú (Figura 35).

Etimología: el nombre específico está dedicado a Luciana Valentina, sobrina del primer autor.

Comentarios: el aparato genital masculino se asemeja al de las especies Epilachna forsteri (Mader) y Epilachna peruviana Crotch, las cuales presentan un hábito con élitros negros y un patrón con dos manchas claras en cada élitro, semejante al de Epilachna matei n.sp. (Figura 24). La placa genital femenina en Epilachna lucianae n.sp. presenta el sexto ventrito fuertemente escotado medialmente, hasta 2/3 de su estructura (figura 08), en comparación a Epilachna peruviana que tiene el sexto ventrito abdominal moderadamente escotado, solo hasta la mitad de su estructura. No se conoce la placa genital de E. forsteri (Gordon 1975).

\section{Epilachna matei $\mathbf{n . s p .}$}

(Figuras 14-30, 35)

Ubicada en el grupo de especies Epilachna vittigera, por presentar el aparato genital masculino con la guía del pene, o lóbulo basal, más larga que los parámeros, con un grupo de setas en cada lado de la parte media, el ápice abruptamente terminado y finamente curvado. Este grupo esta primariamente distribuido en el Perú, con especies extendiéndose a Ecuador, sur de Colombia y norte de Bolivia, siendo el grupo más grande del género y el más diverso en patrones de color y formas (Gordon 1975).

Diagnosis: Coccinélido de color negro, presenta en cada élitro dos manchas redondeadas de color marrón anaranjado a amarillo, la primera mancha cerca de la base y equidistante de ambos bordes, la segunda en el tercio posterior, algo transversa, más cercana al borde lateral que a la sutura. Existen semejanzas con las especies Epilachna dives Erichson y con variaciones de Epilachna fausta Erichson, ambas del grupo flavofasciata, por la conformación de las manchas elitrales; sin embargo, las manchas en dives son más pequeñas y en el caso de la variación de fausta, la mancha posterior, una banda en su forma típica, es muy pequeña y más cercana a la sutura.

Descripción: Macho: largo total $6.3 \mathrm{~mm}$, ancho $4.5 \mathrm{~mm}$, alto $3 \mathrm{~mm}$. Cuerpo corto, acorazonado, dorso fuertemente convexo y densamente pubescente. Cabeza negra. Pronoto totalmente negro. Escutelo negro. Élitros de color negro con 4 manchas amarillas, dos en cada élitro, una anterior, redondeada, cerca de la base y equidistante de los bordes lateral y sutural del élitro, una segunda mancha en el tercio posterior, algo transversa y más cercana del borde lateral. Lado inferior completamente negro, salvo el borde posterior de los ventritos abdominales $2-5$ de color marrón oscuro. Epipleura negra. Patas negras con pubescencia amarillenta, corta, más densa en las tibias.

Cabeza con puntuación fina y densamente distribuida, puntos separados por 1.0 - 1.5 diámetros, asociados con setas claras, largas y dispersas; distancia interocular equivalente a 5.5 veces el ancho del ojo en vista dorsal. Pronoto con puntuación fina, densamente distribuida, más fina que en la cabeza, separada por 1.0 - 2.0 diámetros en el disco pronotal y 1.0 diámetro en los laterales del pronoto. Disco elitral con puntuación fina, similar a la pronotal, separada por 1.0 diámetro a menos. Superficies de metasterno y mesosterno arrugados, con setas cortas claras dispersas. Metaventrito con puntos finos y densamente distribuidos, separados por 1.0 - 1.5 diámetros. Borde posterior del sexto ventrito abdominal moderadamente escotado, hasta casi alcanzar la mitad de la estructura (Figura 22).

Hembra: largo total $7.2 \mathrm{~mm}$, ancho $5.2 \mathrm{~mm}$, alto $4.2 \mathrm{~mm}$. Similar al macho, tamańo algo mayor, las manchas claras del élitro son más grandes y más redondeadas, en especial la mancha posterior que pierde el aspecto transverso (Figuras 27, 28). Borde posterior del sexto ventrito abdominal con escotadura alcanzando un cuarto de su estructura (Figura 23).

Aparato genital masculino: tegmen con la guía del pene, o lóbulo basal, de forma ahusada, más gruesa en el segundo tercio, más largo que los parámeros, presencia de un grupo de setas en la parte lateral media, ápice abruptamente terminado, finamente curvado. Parámeros delgados, con grosor uniforme en toda su extensión, algo engrosada hacia el ápice, ápice redondeado con presencia de setas (Figuras 15 - 18). Pene curvo y delgado, capsula del pene con dos proyecciones, una externa roma dirigida hacia atrás y una interna, roma dirigida hacia el interior, ápice del pene cuadrangular, finamente curvado (Figura 19).

Aparato genital femenino: placa genital femenina con los coxitos ovalados y transversos, sin stylus visible, margen apical con pequeńa protuberancia y presencia de setas (Figura 23). No se estudió la espermateca.

Holotipo: PERU, Machu Picchu, [Machu Picchu, distrito], Urubamba [provincia], Cusco [región], 2040mts, [13⒐52'15”S - 72³3'14.85”O], Feb13 [Febrero 13] 1979, CEUC-UNSAAC [colector].

Alotipo: (hembra) mismos datos que el holotipo.

Paratipos: 10 machos y 12 hembras, mismos datos que el holotipo.

Variación: largo total $6-7.2 \mathrm{~mm}$, ancho $4.5-5.2 \mathrm{~mm}$, alto 


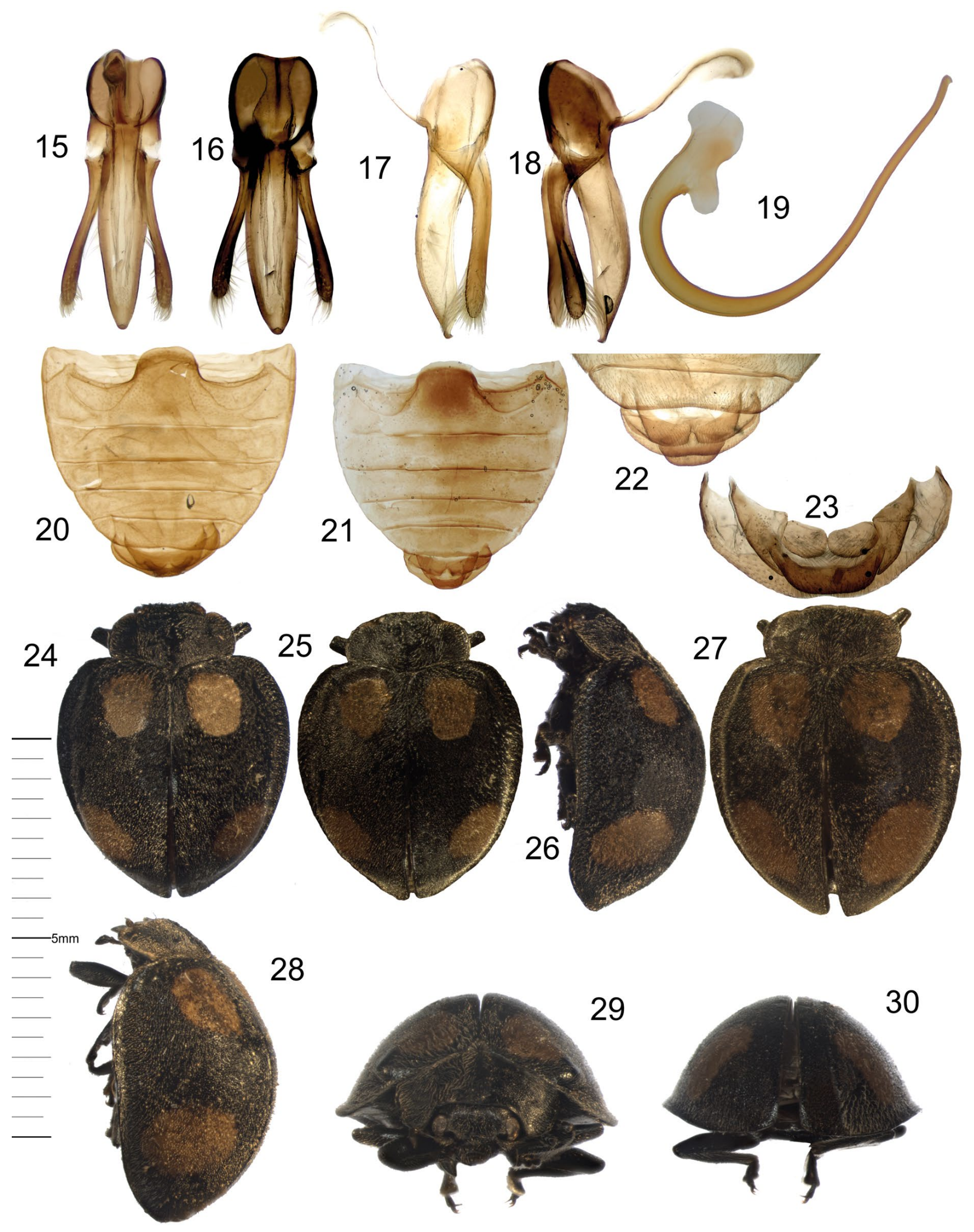

Figuras 15 - 30: Epilachna matei n. sp. (15), (16) Tegmen, vista frontal; (17), (18) Tegmen, vista lateral; (19) Pene, vista lateral; (20) Abdomen, macho; (21) Abdomen, hembra; (22) Urosternitos abdominales, macho; (23) Placa genital, hembra; (24), (25) Hábito macho, vista dorsal; (26) Hábito macho, vista lateral; (27) Hábito hembra, vista dorsal; (28) Hábito hembra, vista lateral; (29) Hábito macho, vista anterior; (30) Habito macho, vista posterior. 


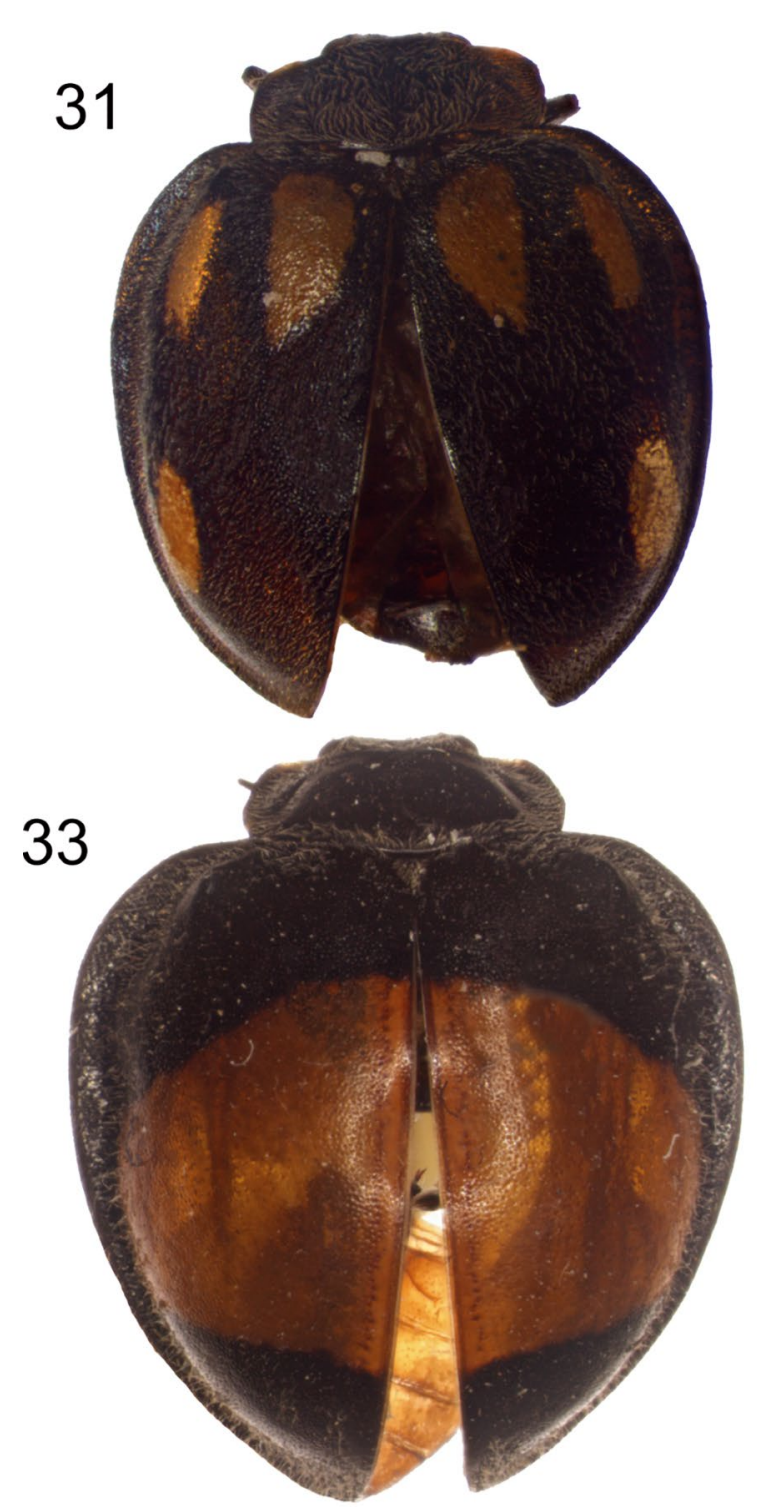

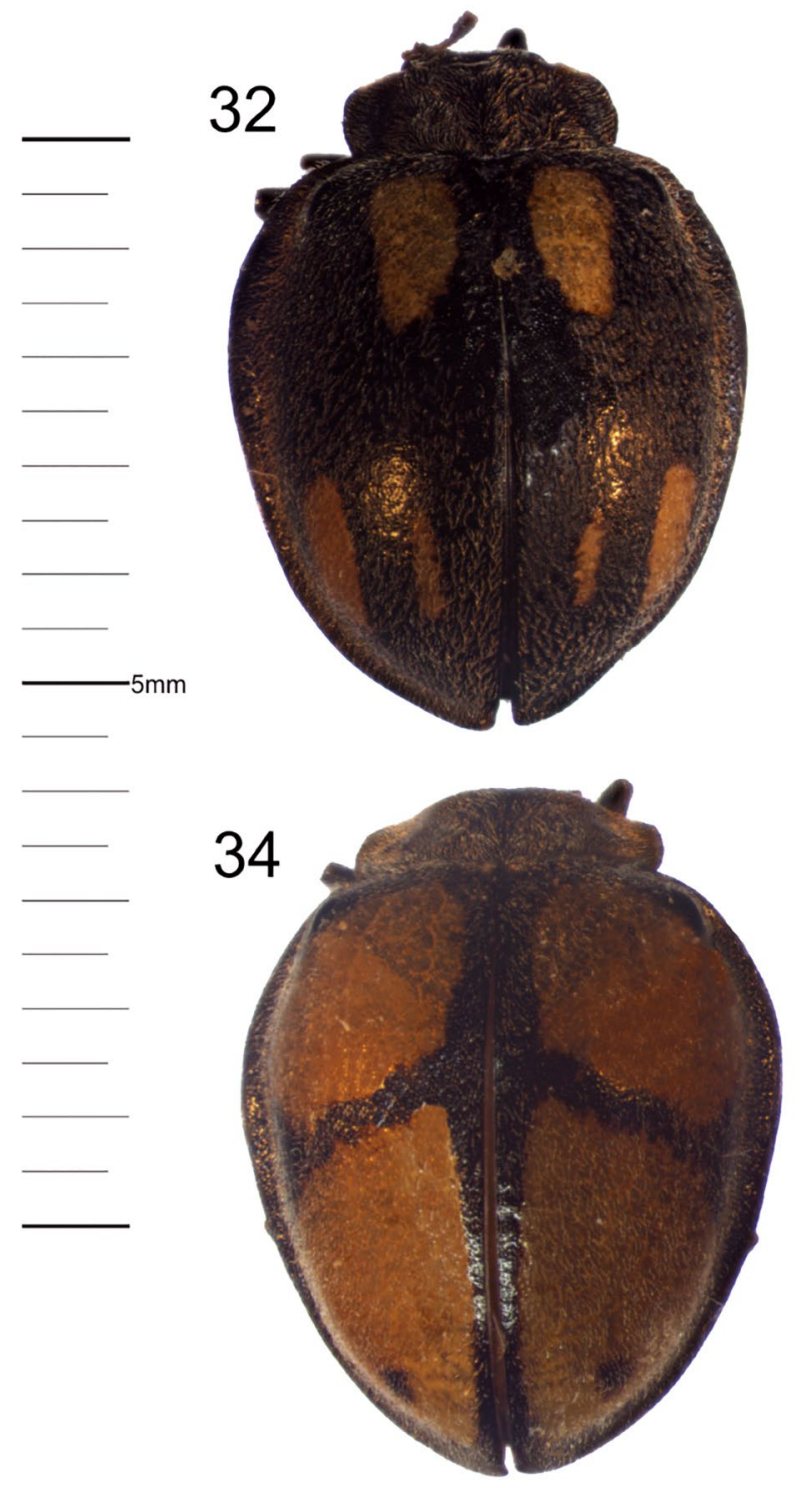

Figuras 31 - 34. (31), (32) Epilachna fenestroides Gordon; (33) Epilachna strictanotata Gordon; (34) Epilachna harringtoni Gordon.

2.8 - $4.2 \mathrm{~mm}$. Algunos ejemplares presentan las manchas del élitro agrandadas y redondeadas.

Distribución: distrito de Machu Picchu, provincia de Urubamba, región Cusco, Perú (Figura 35).

Etimología: el nombre específico está dedicado a Mateo Nicolás, sobrino del primer autor.

Observaciones: si bien la conformación de las manchas elitrales claras de esta especie es semejante a la que presentan algunos ejemplares de Epilachna fausta y E. dives, el aparato genital masculino de $E$. matei, del tipo vittigera, separa a estas especies.

\section{Nuevos registros}

Se presentan registros nuevos, para el Perú, de las especies Epilachna fenestroides Gordon, E. strictanotata Gordon y E. harringtoni Gordon, todas ellas pertenecientes al grupo de especies Epilachna vittigera, por la presencia de setas en la parte media de la guía del pene.

\section{Subfamily: CoccinelLINAE \\ TRIBU: EPILACHNINI GENERO: EPILACHNA CHEVROLAT, 1837 GRUPO: EPILACHNA VITTIGERA}

\section{Epilachna fenestroides Gordon 1975}

(Figuras 31, 32)

Epilachna fenestroides Gordon 1975, p. 104

Material examinado: (15 ejemplares) PERU, Machu Picchu, Urubamba [provincia], Cusco [región], Feb13 [febrero 13] 1979, 2040 mts, CEUC-UNSAAC [colector].

Conocida anteriormente de la localidad tipo, Oxapampa, a 1800 msnm, en la región Pasco, donde esta especie fue encontrada sobre hojas de Baccharis (L) (Gordon 1975). Los especímenes de este nuevo registro corresponden con la variación, manchas elitrales divididas por una banda longitudinal, descrita por Gordon. Nuevo registro regional (Figura 35). 


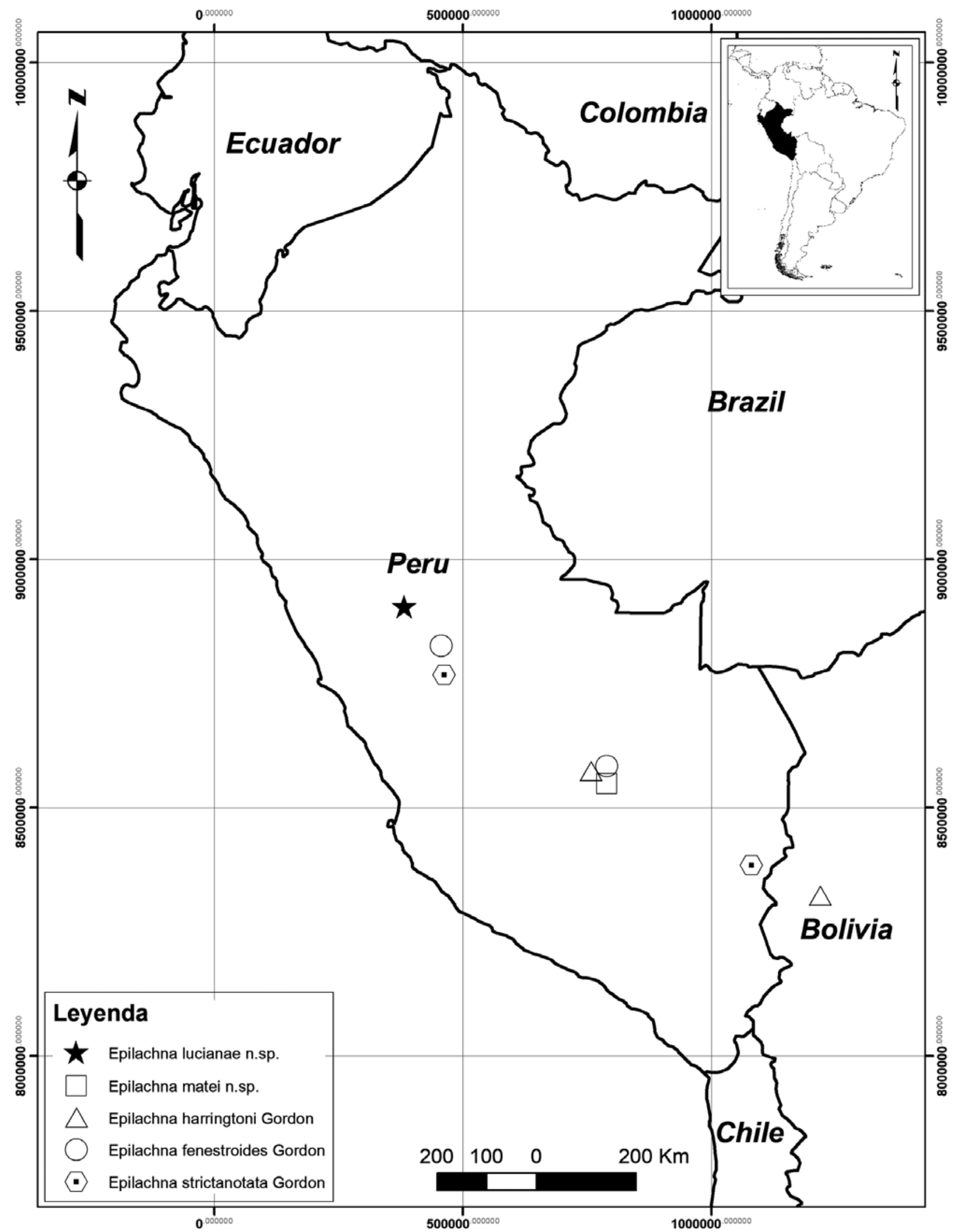

Figura 35. Ubicación de las localidades de colecta de Epilachna lucianae n. sp., Epilachna matei n. sp. y distribución actualizada de Epilachna harringtoni, Epilachna strictanotata y Epilachna fenestroides. 


\section{Epilachna strictanotata Gordon 1975}

(Figura 33)

\section{Epilachna strictanotata Gordon 1975, p. 103}

Material examinado: (1 ejemplar) PERU, Pu [Puno], Sandia, Limbani, Aquele 2110 m, 9-XI-2006 [9 noviembre 2006], J. F. Costa [colector], det. González 2008.

Conocida previamente de la localidad tipo, Chanchamayo, en la región Junín (Gordon 1975), la descripción original indica que la hembra de esta especie es desconocida, lamentablemente el único ejemplar presente en la colección es macho. Nuevo registro regional (Figura 35).

\section{Epilachna harringtoni Gordon 1975}

(Figura 34)

Material examinado: (48 ejemplares) PERU, Machu Picchu, Urubamba [provincia], Cusco [región], Feb13 [febrero 13] 1979, 2040 mts, CEUC-UNSAAC [colector].

Esta especie es conocida solo de la localidad tipo, Mapiri, y Ancapata, ambas en el departamento de La Paz, Bolivia (Gordon 1975). Nuevo registro Perú (Figura 35).

\section{Literatura citada}

Gordon R.D. 1975. A revision of Epilachninae of the Western Hemisphere (Coleoptera: Coccinellidae). Bulletin of the United States Department of Agriculture, Technical Bulletin 1493:1-409.

Ślipiński A. 2007. Australian ladybird beetles (Coleoptera: Coccinellidae). Their biology and classification. ABRS. Camberra, Australia. 286 pp.

Szawaryn K., Tomaszewska W. 2013. Two new genera of Epilachnini Mulsant from New Guinea and Aru Islands (Coleoptera: Coccinellidae). Journal of Natural History, 47:2427-2457. DOI: https://doi.org/10.1080/00222933.2012.763067

Szawaryn, K., L. Bocak, A. Slipinski, H. E. Escalona, and W. Tomaszewska. 2015. Phylogeny and evolution of phytophagous ladybird beetles (Coleoptera: Coccinellidae: Epilachnini), with recognition of new genera. Systematic Entomology, 40:547-569. DOI: https://doi.org/10.1111/syen.12121

Tomaszewska W. \& K. Szawaryn 2016. Epilachnini (Coleoptera: Coccinellidae) - a revision of the world genera. Journal of Insect Science 16(1): 101; 1-91. DOI: https://doi.org/10.1093/ jisesa/iew082 